\title{
Application of distributed point source method (DPSM) to wave propagation in anisotropic media
}

Samaneh Fooladi

Tribikram Kundu 


\title{
Application of Distributed Point Source Method (DPSM) to wave propagation in anisotropic media
}

\author{
Samaneh Fooladi ${ }^{\mathrm{a}}$, Tribikram Kundu ${ }^{* a b}$ \\ ${ }^{a}$ Aerospace and Mechanical Engineering Department, University of Arizona, Tucson, Arizona \\ 85721; ${ }^{\mathrm{b}}$ Department of Civil Engineering and Engineering Mechanics, University of Arizona, \\ Tucson, Arizona 85721
}

\begin{abstract}
Distributed Point Source Method (DPSM) was developed by Placko and Kundu ${ }^{1}$, as a technique for modeling electromagnetic and elastic wave propagation problems. DPSM has been used for modeling ultrasonic, electrostatic and electromagnetic fields scattered by defects and anomalies in a structure. The modeling of such scattered field helps to extract valuable information about the location and type of defects. Therefore, DPSM can be used as an effective tool for Non-Destructive Testing (NDT). Anisotropy adds to the complexity of the problem, both mathematically and computationally. Computation of the Green's function which is used as the fundamental solution in DPSM is considerably more challenging for anisotropic media, and it cannot be reduced to a closed-form solution as is done for isotropic materials. The purpose of this study is to investigate and implement DPSM for an anisotropic medium. While the mathematical formulation and the numerical algorithm will be considered for general anisotropic media, more emphasis will be placed on transversely isotropic materials in the numerical example presented in this paper. The unidirectional fiber-reinforced composites which are widely used in today's industry are good examples of transversely isotropic materials. Development of an effective and accurate NDT method based on these modeling results can be of paramount importance for in-service monitoring of damage in composite structures.
\end{abstract}

Keywords: DPSM, ultrasonic waves, Green's function, anisotropic material

\section{INTRODUCTION}

Distributed Point Source Method (DPSM) is a computational technique for modeling ultrasonic wave problems ${ }^{1,2}$. The scattering of ultrasonic acoustic waves by defects is important in Non-Destructive Testing and evaluation (NDT\&E). For ultrasonic NDE (non-destructive evaluation) of a solid plate, it is a common practice to immerse the plate in a fluid. The ultrasonic waves are transferred from the transducer to the fluid and then from the fluid to the solid.

DPSM has been used for modeling ultrasonic waves in a homogenous isotropic solid plate immersed in a fluid ${ }^{1,3}$. A finite-size scatterer can be added to this problem to represent the problem of scattering by an internal anomaly in the plate $^{1}$. Later, a circular cylindrical hole was added to a solid half-space ${ }^{3}$, and an elliptical cavity was considered ${ }^{4}$. The interface between fluid and solid can have an arbitrary shape in general. Therefore, non-planar and relatively complex geometries for solid-fluid interface should be also considered, and have been modeled by DPSM ${ }^{5,6,7}$. In all of those studies the solid is assumed to be homogeneous and isotropic for which the Green's functions are readily available as a closed-form expression.

In contrast to isotropic materials, the Green's function for anisotropic materials cannot be expressed as a closed-from solution. This adds to the complexity of the problem both mathematically and computationally. For an anisotropic material, the elastodynamic Green's function should be evaluated numerically. Wang and Achenbach ${ }^{8,9}$ formulated elastodynamic time-harmonic Green's functions for anisotropic solids using Radon transform. Their solution method is adopted in this paper. In addition, the singular part of the solution is evaluated by the calculus of residue ${ }^{10,11}$. The Green's function calculations are then used as the building blocks in a DPSM model. The developed anisotropic DPSM model is used to simulate ultrasonic waves in a composite plate immersed in fluid. The transducers are placed in fluid on both sides of the plate.

*Corresponding author: tkundu@email.arizona.edu; phone 1520 621-6573;

Health Monitoring of Structural and Biological Systems 2017, edited by Tribikram Kundu, Proc. of SPIE Vol. 10170, 101700P · (c) 2017 SPIE · CCC code: 0277-786X/17/\$18 · doi: 10.1117/12.2258573 
The remainder of this paper is organized in the following manner. Section 2 briefly reviews the DPSM formulation for a solid plate immersed in fluid. In section 3, the formulation of elastodynamic Green's function is briefly presented. Next in section 4, the numerical results for a transversely isotropic material are shown. Lastly, in section 5 the conclusion is given.

\section{DPSM FOR A SOLID PLATE IMMERSED IN FLUID}

DPSM has been previously formulated for a solid plate immersed in fluid ${ }^{1,3}$. It is briefly reviewed in this section. Let us consider a solid plate in contact with fluid, as shown in figure 1. A flat square transducer called transducer 1 is placed inside fluid 1. Similarly, transducer 2 is placed inside fluid 2. A collection of source points are distributed near the boundaries. In Figure 1, only the point sources on a vertical plane are plotted for the sake of clarity. Different sets of point sources are defined in Table 1.

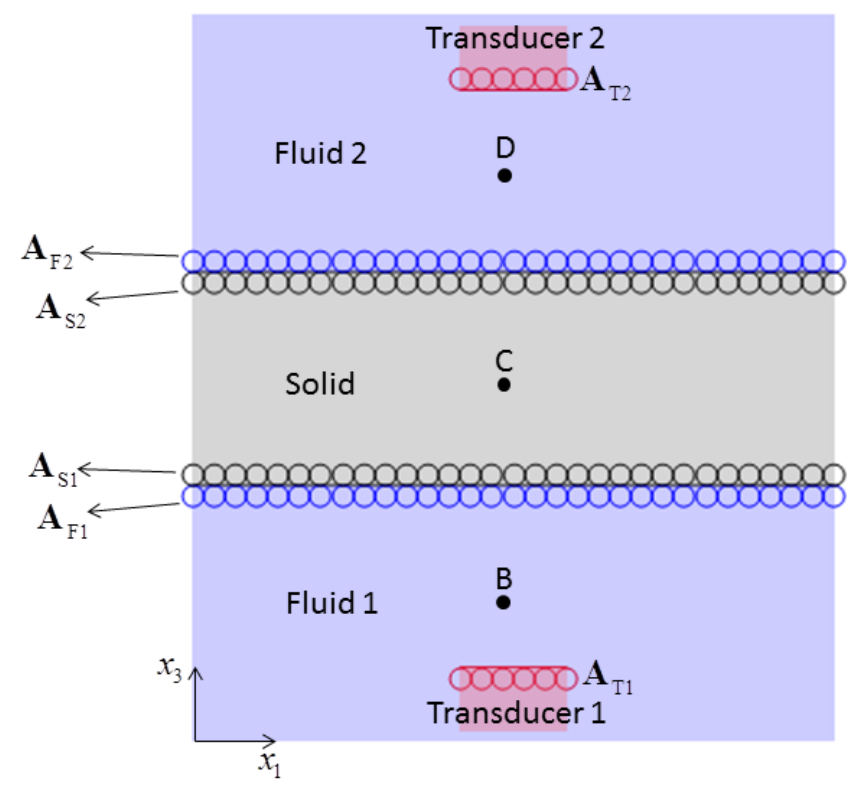

Figure 1. Problem description and distribution of point sources.

Table 1 . The list of source strength vectors.

\begin{tabular}{|c|l|}
\hline $\begin{array}{c}\text { Source strength } \\
\text { vector }\end{array}$ & Location of corresponding point sources \\
\hline $\mathbf{A}_{\mathrm{T} 1}$ & Inside transducer 1 and near its surface \\
\hline $\mathbf{A}_{\mathrm{T} 2}$ & Inside transducer 2 and near its surface \\
\hline $\mathbf{A}_{\mathrm{F} 1}$ & Inside fluid 1 and near its interface with the solid \\
\hline $\mathbf{A}_{\mathrm{F} 2}$ & Inside fluid 2 and near its interface with the solid \\
\hline $\mathbf{A}_{\mathrm{S} 1}$ & Inside solid and near its interface with fluid 1 \\
\hline $\mathbf{A}_{\mathrm{S} 2}$ & Inside solid and near its interface with fluid 2 \\
\hline
\end{tabular}


Next, the boundary and interface conditions are applied in order to obtain the values of source strength vectors listed in Table 1. Let us place a collection of target points on the surface of transducer 1 and denote them as $\mathrm{J} 1$, and on the surface of transducer 2 and denote them as $\mathrm{J} 2$. Then, the components of velocity vector normal to the surface of transducer can be written as

$$
\mathbf{V}_{\mathrm{J} 1}=\mathbf{M}_{\mathrm{J} 1, \mathrm{~T} 1}^{V_{3}} \mathbf{A}_{\mathrm{T} 1}+\mathbf{M}_{\mathrm{J} 1, \mathrm{~S} 1}^{V_{3}} \mathbf{A}_{\mathrm{S} 1}
$$

and

$$
\mathbf{V}_{\mathrm{J} 2}=\mathbf{M}_{\mathrm{J} 2, \mathrm{~T} 2}^{V_{3}} \mathbf{A}_{\mathrm{T} 2}+\mathbf{M}_{\mathrm{J} 2, \mathrm{~S} 2}^{V_{3}} \mathbf{A}_{\mathrm{S} 2}
$$

where the array $\mathbf{M}_{\mathrm{T}, \mathrm{S}}^{q}$ is constructed based on the information from the Green's function of source point(s) $\mathrm{S}$ at target point(s) $\mathrm{T}$, for quantity $q$. At each of the two fluid-solid interfaces, the normal displacement and normal stress are continuous, and the shear stress is zero due to the assumption of non-viscous fluid. Let us place a collection of target points on the lower interface and denote them by I1, and on the upper interface and denote them by $\mathrm{I} 2$. Then, the continuity conditions on the lower interface give

$$
\begin{aligned}
& \mathbf{M}_{\mathrm{I} 1, \mathrm{~T} 1}^{U_{3}} \mathbf{A}_{\mathrm{T} 1}+\mathbf{M}_{\mathrm{I} 1, \mathrm{~S} 1}^{U_{3}} \mathbf{A}_{\mathrm{S} 1}=\mathbf{M}_{\mathrm{I} 1, \mathrm{~F} 1}^{U_{3}} \mathbf{A}_{\mathrm{F} 1}+\mathbf{M}_{\mathrm{I} 1, \mathrm{~F} 2}^{U_{3}} \mathbf{A}_{\mathrm{F} 2} \\
& \mathbf{M}_{\mathrm{I} 1, \mathrm{~T} 1}^{P} \mathbf{A}_{\mathrm{T} 1}+\mathbf{M}_{\mathrm{I} 1, \mathrm{~S} 1}^{P} \mathbf{A}_{\mathrm{S} 1}=-\mathbf{M}_{\mathrm{I} 1, \mathrm{~F} 1}^{S_{33}} \mathbf{A}_{\mathrm{F} 1}-\mathbf{M}_{\mathrm{I} 1, \mathrm{~F} 2}^{S_{33}} \mathbf{A}_{\mathrm{F} 2} \\
& \mathbf{M}_{\mathrm{I} 1, \mathrm{~F} 1}^{S_{31}} \mathbf{A}_{\mathrm{F} 1}+\mathbf{M}_{\mathrm{I} 1, \mathrm{~F} 2}^{S_{31}} \mathbf{A}_{\mathrm{F} 2}=0 \\
& \mathbf{M}_{\mathrm{I} 1, \mathrm{~F} 1}^{S_{32}} \mathbf{A}_{\mathrm{F} 1}+\mathbf{M}_{\mathrm{I} 1, \mathrm{~F} 2}^{S_{32}} \mathbf{A}_{\mathrm{F} 2}=0
\end{aligned}
$$

Similarly the continuity conditions on the upper interface give

$$
\begin{aligned}
& \mathbf{M}_{\mathrm{I} 2, \mathrm{~T} 2}^{U_{3}} \mathbf{A}_{\mathrm{T} 2}+\mathbf{M}_{\mathrm{I} 2, \mathrm{~S} 2}^{U_{3}} \mathbf{A}_{\mathrm{S} 2}=\mathbf{M}_{\mathrm{I} 2, \mathrm{~F} 1}^{U_{3}} \mathbf{A}_{\mathrm{F} 1}+\mathbf{M}_{\mathrm{I} 2, \mathrm{~F} 2}^{U_{3}} \mathbf{A}_{\mathrm{F} 2} \\
& \mathbf{M}_{\mathrm{I} 2, \mathrm{~T} 2}^{P} \mathbf{A}_{\mathrm{T} 2}+\mathbf{M}_{\mathrm{I} 2, \mathrm{~S} 2}^{P} \mathbf{A}_{\mathrm{S} 2}=-\mathbf{M}_{\mathrm{I} 2, \mathrm{~F} 1}^{S_{33}} \mathbf{A}_{\mathrm{F} 1}-\mathbf{M}_{\mathrm{I} 2, \mathrm{~F} 2}^{S_{33}} \mathbf{A}_{\mathrm{F} 2} \\
& \mathbf{M}_{\mathrm{I} 2, \mathrm{~F} 1}^{S_{31}} \mathbf{A}_{\mathrm{F} 1}+\mathbf{M}_{\mathrm{I} 2, \mathrm{~F} 2}^{S_{31}} \mathbf{A}_{\mathrm{F} 2}=0 \\
& \mathbf{M}_{\mathrm{I} 2, \mathrm{~F} 1}^{S_{32}} \mathbf{A}_{\mathrm{F} 1}+\mathbf{M}_{\mathrm{I} 2, \mathrm{~F} 2}^{S_{32}} \mathbf{A}_{\mathrm{F} 2}=0
\end{aligned}
$$

where $U_{i}$ represents component $i$ of the displacement vector, $P$ is the pressure, and $S_{i j}$ represents $(i, j)$ component of the stress tensor. Simultaneous solution of equations (1), (2), (3a-d), and (4a-d), gives the values of all source strength vectors listed in Table 1. After finding the source strengths, the solution at any target point can be computed.

\section{THE GREEN'S FUNCTION FOR ANISOTROPIC MATERIALS}

In this section, the derivation of the Green's function for anisotropic materials presented by Wang and Achenbach ${ }^{8,9}$ is briefly reviewed. The equations of motion in an anisotropic homogenous medium can be written as

$$
C_{i j k l} \frac{\partial^{2} u_{k}}{\partial x_{j} \partial x_{l}}+f_{i}=\rho \frac{\partial^{2} u_{i}}{\partial t^{2}}
$$


where $C_{i j k l}$ are the components of the stiffness tensor, $u_{i}$ are the components of the displacement vector, $f_{i}$ are the components of the body force density vector, and $\rho$ is the mass density. The elastodynamic Green`s function $\mathbf{G}\left(\mathbf{x}, \mathbf{x}_{0}\right)$ gives the displacement at point $\mathbf{x}$ when a point force is applied at point $\mathbf{x}_{0}$. Assuming a time harmonic force $f_{i}(\mathbf{x}, t)=\delta_{i p} \delta\left(\mathbf{x}-\mathbf{x}_{0}\right) e^{-\mathbf{i} \omega t}$ and a time harmonic displacement $u_{i}(\mathbf{x}, t)=G_{i p}\left(\mathbf{x}, \mathbf{x}_{0}\right) e^{-\mathbf{i} \omega t}$, the equation (5) is converted to

$C_{i j k l} \frac{\partial^{2} G_{k p}\left(\mathbf{x}, \mathbf{x}_{0}\right)}{\partial x_{j} \partial x_{l}}+\rho \omega^{2} G_{i p}\left(\mathbf{x}, \mathbf{x}_{0}\right)=-\delta_{i p} \delta\left(\mathbf{x}-\mathbf{x}_{0}\right)$

The above equation can be solved using Radon transform. Let $f(\mathbf{x})$ be an arbitrary function in 3D space. The Radon transform of this function $\mathfrak{R}[f(\mathbf{x})]$ is defined as

$\hat{f}(s, \mathbf{n})=\mathfrak{R}[f(\mathbf{x})]=\int f(\mathbf{x}) \delta(s-(\mathbf{n} \cdot \mathbf{x})) d \mathbf{x}$

where $\mathbf{n}$ is a unit vector. The inverse of Radon transform is

$f(\mathbf{x})=\mathfrak{R}^{-1}[f(\mathbf{x})]=-\frac{1}{8 \pi^{2}} \int_{|\mathbf{n}|=1} \frac{\partial^{2} \hat{f}(s, \mathbf{n})}{\partial s^{2}} d S(\mathbf{n})$

By applying Radon transform, the equation (6) is reduced to

$$
\left(K_{i k}(\mathbf{n}) \frac{\partial^{2}}{\partial s^{2}}+\rho \omega^{2} \delta_{i k}\right) \hat{G}_{k p}=-\delta_{i p} \delta(s)
$$

where $K_{i k}(\mathbf{n})=C_{i j k l} n_{l} n_{j}$. By transforming the coordinates to a new set of bases formed by eigenvectors of $K_{i k}(\mathbf{n})$ the above equation is uncoupled. The result is

$$
\left.\left(\lambda_{m} \frac{\partial^{2}}{\partial s^{2}}+\rho \omega^{2}\right) \hat{G}_{m q}^{*}=-\delta_{m q} \delta(s), \quad m=1,2,3 \text { (no sum on } m\right)
$$

where $\lambda_{m}(m=1,2,3)$ are eigenvalues of $\mathbf{K}$. The solution to the above equation can be written as

$\hat{G}_{m q}^{*}=\frac{\mathbf{i} \delta_{m q}}{2 \rho c_{m}^{2} \alpha_{m}} e^{\mathbf{i} \alpha_{m}|s|}$

where $c_{m}=\sqrt{\lambda_{m} / \rho}$ and $\alpha_{m}=\omega / c_{m}$ are phase velocity and wave number, respectively. The solution in equation (11) is transformed back to the original set of basis to give

$$
\hat{G}_{k p}=\frac{\mathbf{i} E_{k m} E_{p m}}{2 \rho c_{m}^{2} \alpha_{m}} e^{\mathbf{i} \alpha_{m}|s|}
$$

where $\mathbf{E}$ is a matrix with columns representing eigenvectors of $\mathbf{K}$. Finally, applying the inverse Radon transform to the above expression gives $G_{k p}\left(\mathbf{x}, \mathbf{x}_{0}\right)$ as 
$G_{k p}^{S}\left(\mathbf{x}, \mathbf{x}_{0}\right)=\frac{\mathbf{i}}{8 \pi^{2}} \int_{|\mathbf{n}|=1} \frac{\alpha_{m} E_{k m} E_{p m}}{\rho c_{m}^{2}} e^{\mathbf{i} \alpha_{m}\left|\mathbf{n} \cdot\left(\mathbf{x}-\mathbf{x}_{0}\right)\right|} d A(\mathbf{n})+\frac{1}{8 \pi^{2} r} \oint_{S} K_{k p}^{-1}(\xi) d S(\xi)$

where $K_{i k}(\xi)=C_{i j k l} \xi_{l} \xi_{j}$ and $S$ is an inclined circle on a unit sphere.

\section{NUMERICAL EXAMPLE}

A transversely isotropic plate is considered. The problem geometry is shown in Figure 1. The thickness of the fluid layer is $5 \mathrm{~mm}$ for both upper and lower layers. The thickness of the plate is also $5 \mathrm{~mm}$. The transducers have square shape with edge length of $2 \mathrm{~mm}$, and are oriented so that the axis $x_{3}$ is normal to their faces. A velocity amplitude $V=1 \mathrm{~m} / \mathrm{s}$ and a frequency $f=500 \mathrm{kHz}$ are assumed for both transducers. The fluid is assumed to be water with density $\rho=1000 \mathrm{~kg} / \mathrm{m}^{3}$ and P-wave speed $C_{p}=1480 \mathrm{~m} / \mathrm{s}$. The solid plate is assumed to be Graphite-epoxy composite. The stiffness tensor and density for the solid plate are ${ }^{12}$

$\rho=1610 \mathrm{~kg} / \mathrm{m}^{3}$

and

$\mathbf{C}=\left[\begin{array}{llllll}161 & 6.5 & 6.5 & & & \\ 6.5 & 14.5 & 7.24 & & & \\ 6.5 & 7.24 & 14.5 & & & \\ & & & 3.63 & & \\ & & & & 7.1 & \\ & & & & & 7.1\end{array}\right] \mathrm{GPa}$

respectively. The $x_{1} x_{3}$ plane passing through $x_{2}=L_{2} / 2$ is considered. The results for stress distribution on this plane are shown in Figure 2. Next, the $x_{2} x_{3}$ plane passing through $x_{1}=L_{1} / 2$ is considered. The results for stress distribution on this plane are shown in Figure 3. The anisotropic behavior of the plate becomes evident when one compares the stress values on $x_{1} x_{3}$ plane shown in Figure 2 with those on $x_{2} x_{3}$ plane shown in Figure 3. 


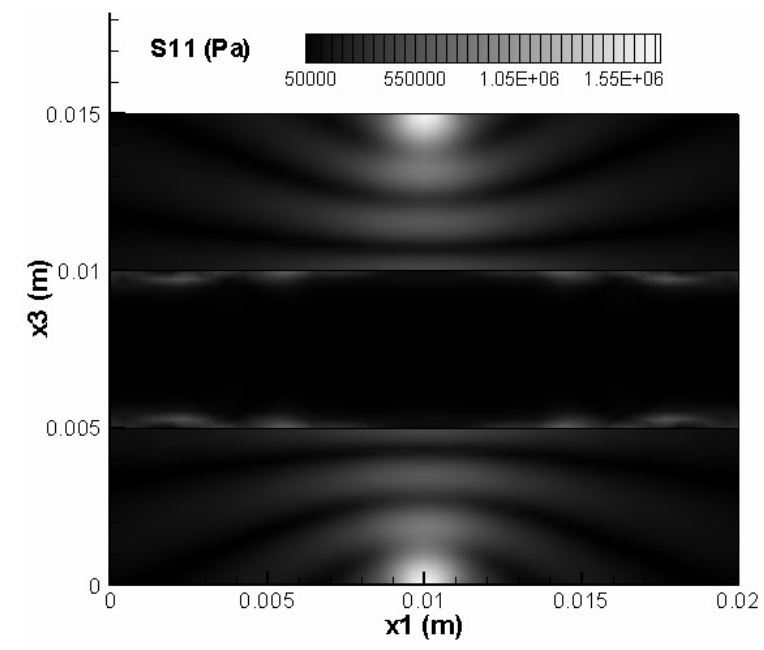

(a)

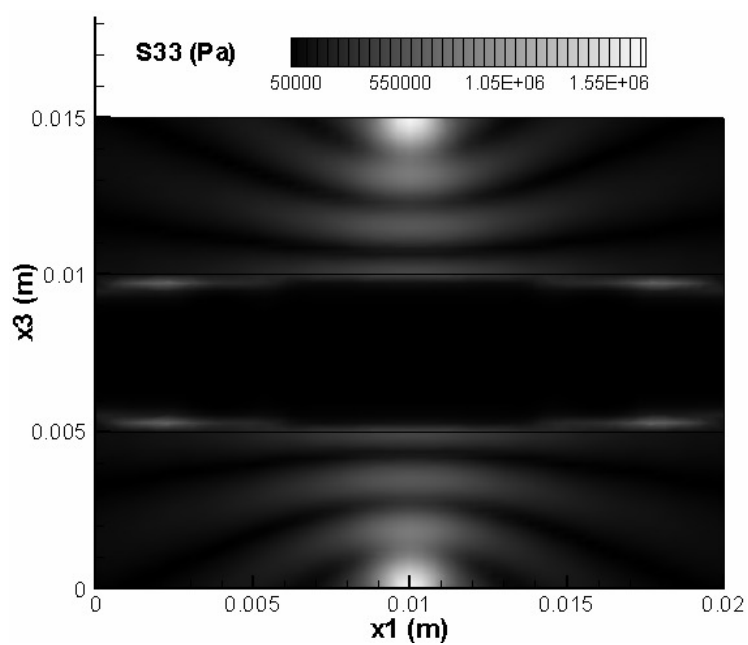

(c)

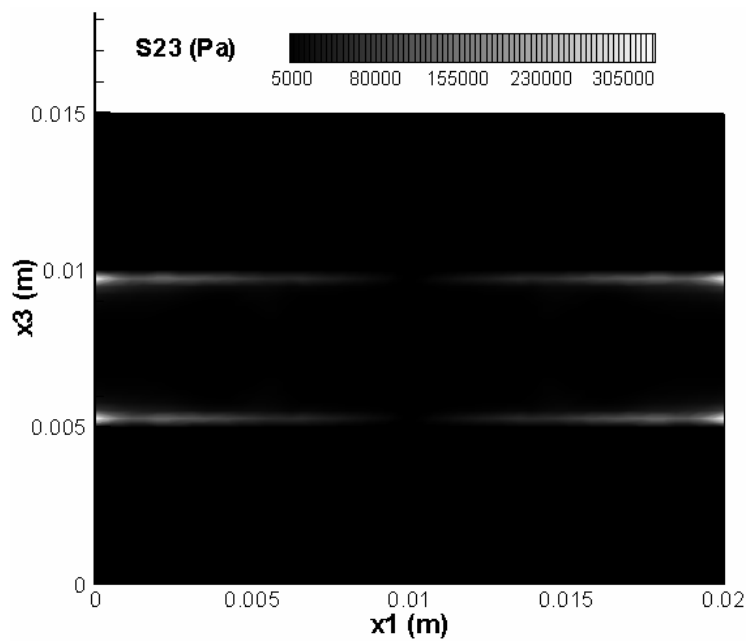

(e)

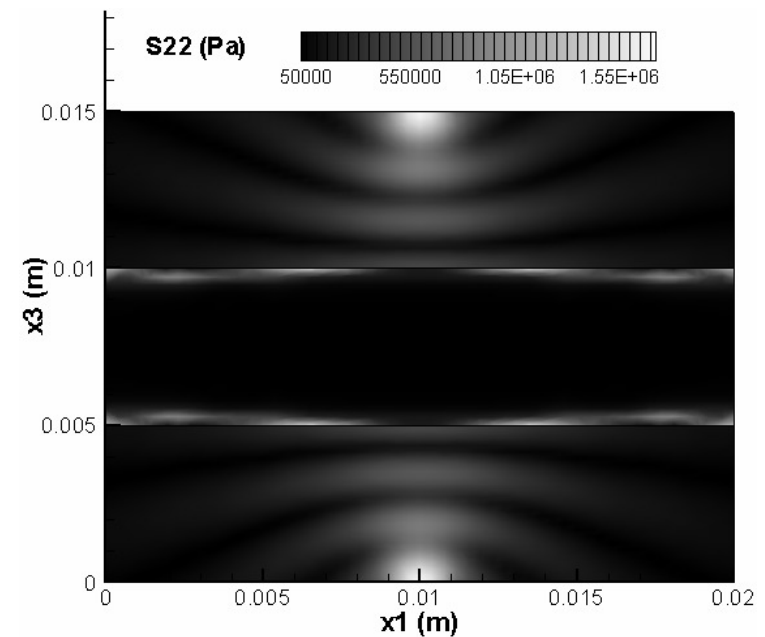

(b)

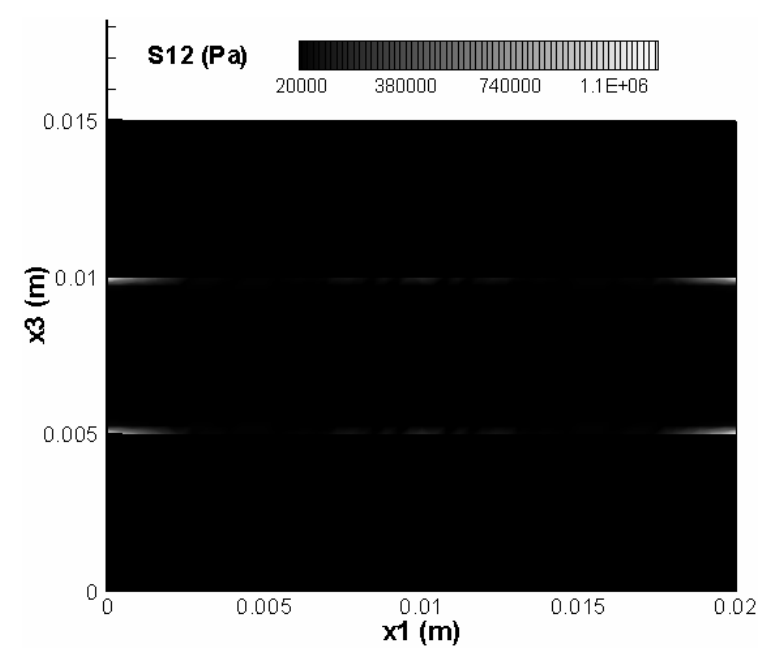

(d)

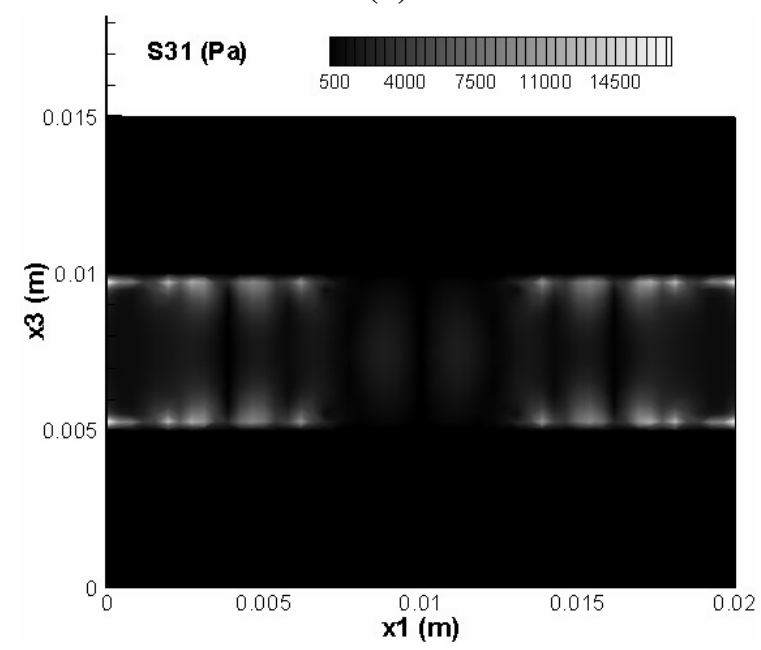

(f)

Figure 2. Anisotropic plate: The stress variations at the central $x_{1} x_{3}$ plane. 


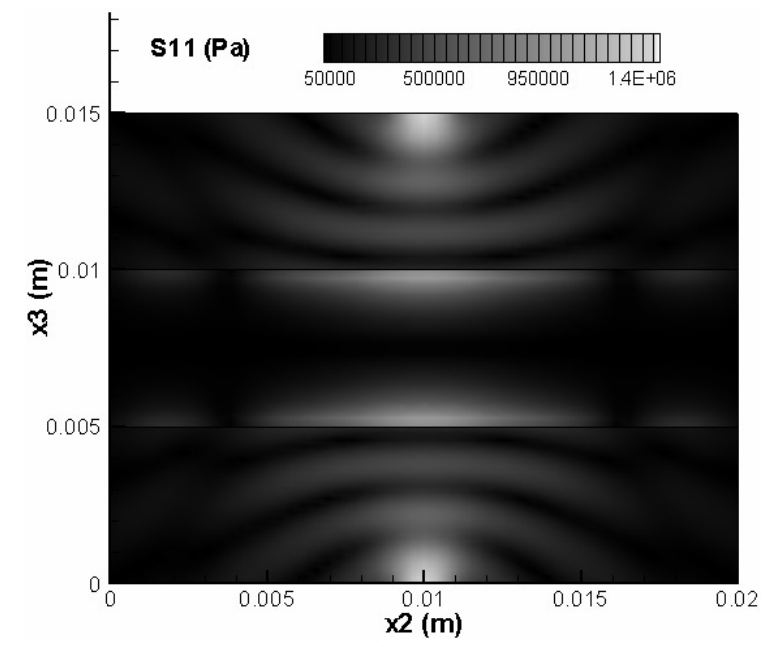

(a)

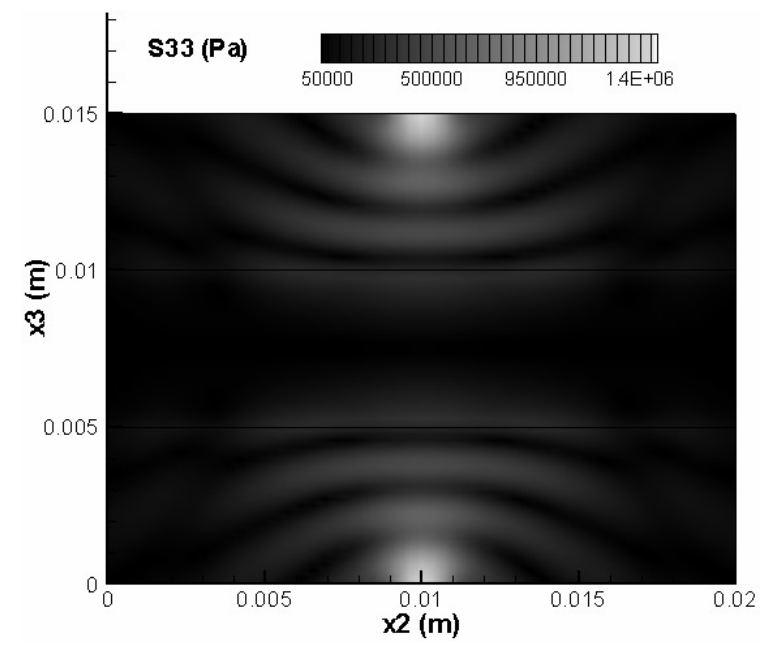

(c)

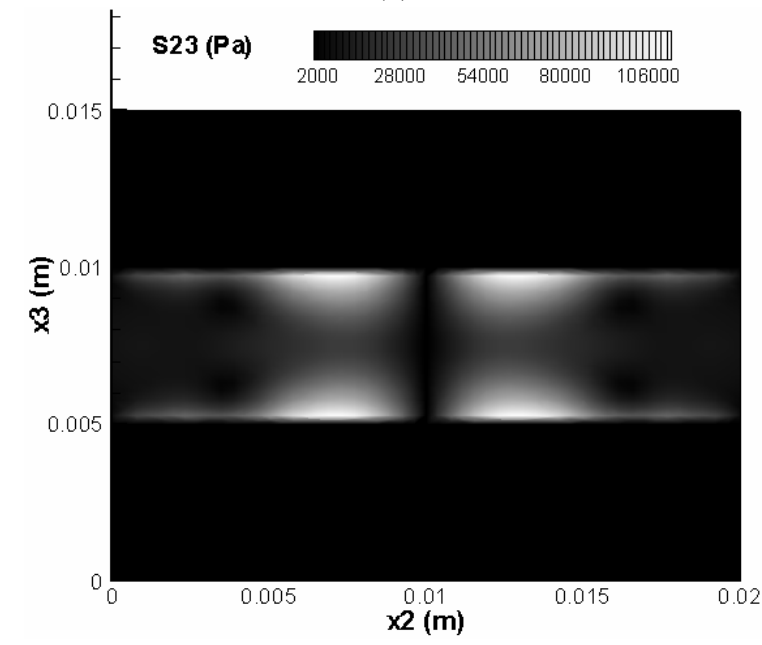

(e)

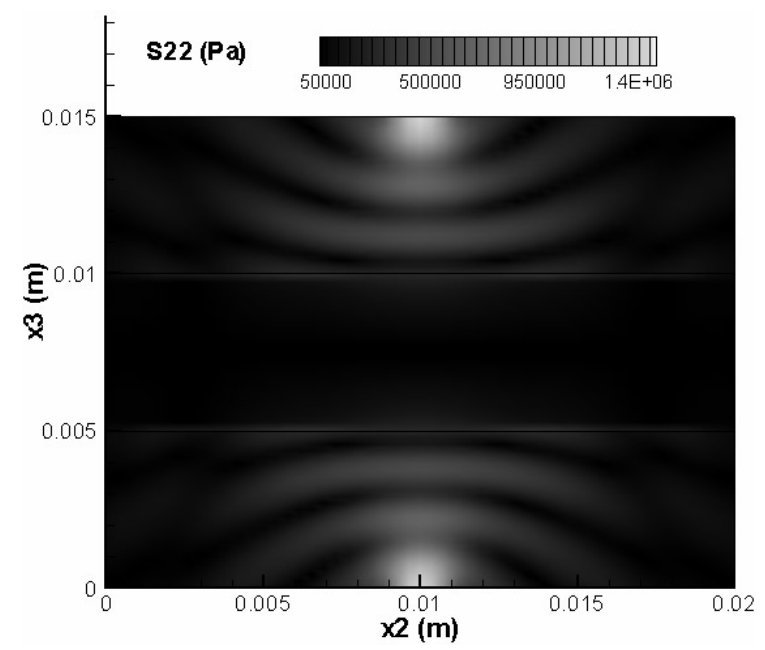

(b)

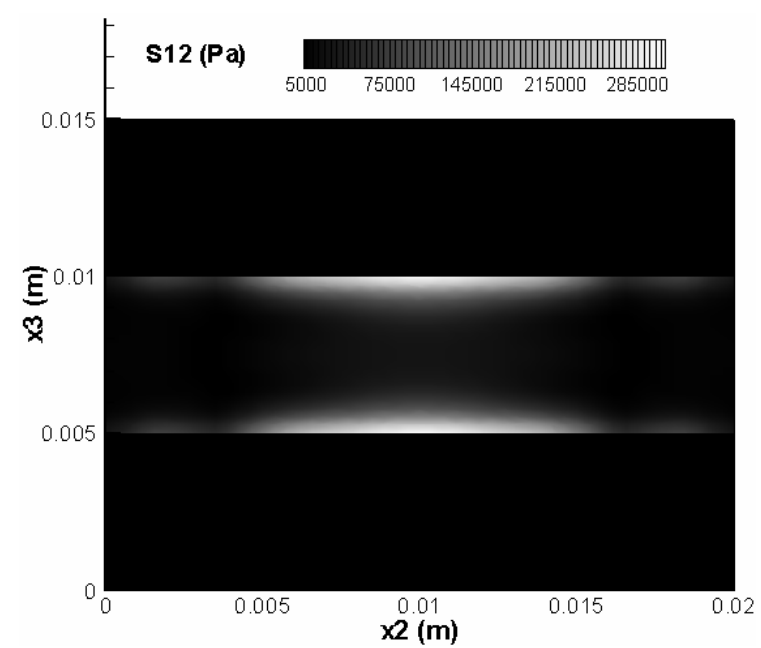

(d)

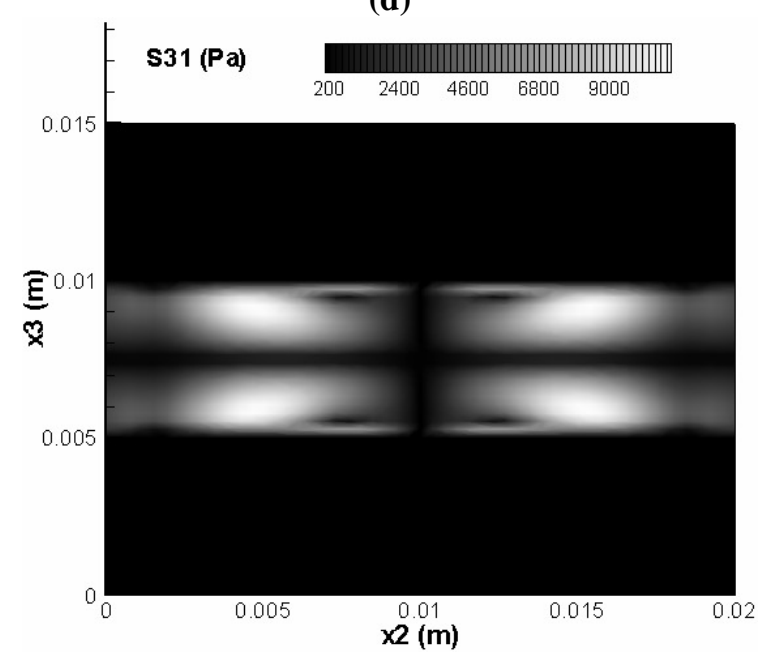

(f)

Figure 3. Anisotropic plate: The stress variations at the central $x_{2} x_{3}$ plane. 


\section{CONCLUSIONS}

The application of DPSM for ultrasonic wave propagation modeling in anisotropic materials has been considered in this study. The anisotropic Green's function was evaluated numerically. The developed anisotropic DPSM was used to simulate ultrasonic wave propagation in a solid plate immersed in fluid with transducers on both sides of the plate. A transversely isotropic plate made of Graphite-epoxy was considered and the developed anisotropic DPSM model was applied to solve this problem.

\section{REFERENCES}

[1] Placko, D., and Kundu, T., Eds. “DPSM for Modeling Engineering Problems,” Hoboken, NJ: Wiley (2007).

[2] Kundu, T., Placko, D., Rahani, E.K., Yanagita, T., and Dao, C.M., "Ultrasonic Field Modeling: A Comparison between Analytical, Semi-Analytical and Numerical Techniques," IEEE Transactions on Ultrasonics, Ferroelectric and Frequency Control 57(12), 2795-2807 (2010).

[3] Banerjee, S. and Kundu, T., "Ultrasonic Field Modelling in Plates immersed in Fluid," International Journal of Solids and Structures 44, 6013-6029 (2007).

[4] Das, S., Banerjee, S., and Kundu, T., "Elastic wave scattering in a solid half-space with a circular cylindrical hole using the Distributed Point Source Method," International Journal of Solids and Structures 45, 4498-4508 (2008).

[5] Shelke, A., Das, S., and Kundu, T., "Distirbuted point source method for modeling scattered ultrasonic fields in the presence of an elliptical cavity," Structural health monitoring 9(6), 527-539 (2010).

[6] Das, S., Dao, C.M., Banerjee, S., and Kundu, T., "DPSM modeling for studying interaction between bounded ultrasonic beams and corrugated plates," IEEE Trans. Ultrason. Ferroelectr. Freq. Control, 54(9), 1860-1872 (2007).

[7] Banerjee, S., and Kundu, T., "Elastic Wave Field Computation in Multilayered Non-Planar Solid Structures: A MeshFree Semi-Analytical Approach," Journal of the Acoustical Society of America, 123(3), 1371-1382 (2008).

[8] Wang, C.Y., and Achenbach, J.D., "Elastodynamic fundamental solutions for anisotropic solids," Geophys. J. Int. (118), 384-392 (1994).

[9] Wang, C.Y., and Achenbach, J.D., "Three-dimensional time-harmonic elastodynamic Green's functions for anisotropic solids," Proceedings of the Royal Society A 449, 441-458 (1995).

[10] Sales, M.A., Gray, L.J., "Evaluation of the anisotropic Green's function and its derivatives," Computers and Structures 69, 247-254 (1998).

[11] Dederichs, P.H., and Liebfried, G., "Elastic Green's function for anisotropic cubic crystals," Physical Review 188, 1175-83 (1969).

[12] Kriz, R.D., and Stinchcomb, W.W., "Elastic moduli of transversely isotropic Graphite fibers and their composites," Experimental Mechanics, 41-49 (1978). 